\title{
Exercícios físicos durante a gestação: motivos da não adesão
}

Physical exercises during pregnancy: reasons for non-adherence

\author{
Ana Luisa Batista Santos \\ Universidade Estadual do Ceará, Brasil \\ luisa.batista@uece.br
}

\section{Luilma Albuquerque Gurgel \\ Universidade Estadual do Ceará, Brasil \\ luilma.gurgel@uece.br}

\author{
Alba Menezes de Freitas Neta \\ Universidade Estadual do Ceará, Brasil \\ albafreitas.ef@gmail.com
}

\section{Resumo:}

Objetivou-se identificar os motivos da não adesão à prática de exercício físico durante a gestação, para que os profissionais de saúde envolvidos na assistência pré-natal possam identificar e intervir na atenuação desses fatores limitantes. Trata-se de uma revisão integrativa, cujos critérios de inclusão foram artigos disponíveis na íntegra on line nos idiomas português, inglês e espanhol, publicados e indexados nas bases de dados Scielo, Medline, Lilacs, Cochrane Library e Science Direct, no período de 2006 a 2016, e que apresentassem variáveis relacionadas à não adesão à prática de exercício físico durante a gestação de risco habitual. Foram analisados 56 artigos, dos quais se extraíram evidências classificadas em fatores intrapessoais, processos interpessoais, fatores ambientais e políticas públicas. Predominaram achados relacionados aos fatores intrapessoais e processos interpessoais. Esse fato indica que o maior número de barreiras concerne a elementos relacionados intrinsicamente à gestante e às relações construídas com conjuge, familiares, amigos, profissionais de saúde e sociedade com os quais a gestante convive, foi educada e interage cotidianamente.

Palavras-chave: Gestantes, Exercício, Cuidado Pré-Natal, Atenção Primária à Saúde, Saúde Pública.

\section{ABSTRACT:}

This study aimed to identify the reasons for the practice of physical exercise during pregnancy, so that health professionals who participate in prenatal practice can be able to identify and attenuate these limiting factors. This is an integrative review, whose selection criteria were full-text papers available online in Portuguese, English and Spanish; published and indexed in databases Scielo, Medline, Lilacs, Cochrane Library and Science Direct databases, in the period 2006-2016, that presented the variables related to the lack of physical exercise during the habitual risk gestation. Fitfy-six papers were analyzed, from which were retrieved variables classified as intrapersonal factors, interpersonal processes, environmental factors, and public policies. Findings related to intrapersonal factors and interpersonal processes predominated. This fact indicates the greater amount of barriers is related to an element intrinsically related to the pregnant women and their relations with family members, friends, health professionals, and society, with who they live, has been educated and interact daily.

KEYworDs: Pregnant women, Exercise, Prenatal Care, Primary Health Care, Public Health.

\section{INTRODUÇÃo}

O exercício físico é caracterizado como toda atividade física estruturada, planejada e repetitiva que tem por objetivo a melhoria da saúde e a manutenção da aptidão física, sendo uma subcategoria da atividade física (Caspersen, Powell \& Christenson, 1985). Sua prática pode e deve ser recomendada para todas as gestantes saudáveis (Nascimento, Godoy, Surita \& Silva, 2014). É classificada como gestante fisicamente ativa aquelas que praticam exercício de 4 e 5 vezes na semana em sessões de 30 minutos ou mais, não 
ultrapassando 60 minutos contínuos (American College of Obstetricians and Gynecologists, 2002, Wolfe \& Davies, 2003, Zarvosky \& Longo, 2011).

Evidências crescentes sugerem que o exercício desempenha papel na redução do risco de complicações na gravidez, como pré-eclâmpsia, parto prematuro, diabetes gestacional, pode ajudar a prevenir o ganho de peso excessivo na gravidez e a retenção de peso no pós-parto (Fell, Joseph, Armson \& Dodds, 2008). Ademais, os benefícios da prática na prevenção de doenças e no fortalecimento da musculatura recrutada durante o parto normal estão intimamente relacionados com a redução das taxas de morbi-mortalidade materno infantil (Dempsey, Butler \& Williams, 2004, Fell et al., 2008, Garshasbi \& Faghih Zadeh, 2005, Goodwin, Astbury \& Mcmeeken, 2000, Kihlstrand, Stenman, Nilsson \& Axelsson, 1999, Marquez-Sterling, Kaplan, Halberstein \& Signorile, 2000, Poudevigne, O'Connor, 2006, 2005, Yeo, Steele, Chang, Leclaire, Ronis \& Hayashi, 2000).

A Política Nacional de Promoção a Saúde (PNPS) determinou a prática corporal/atividade física como um eixo de ação na saúde pública brasileira (Brasil, 2006). Contudo, estudos realizados no Brasil mostram que a porcentagem de gestantes fisicamente inativas ainda é alta e demonstram que esse tema deve ser melhor abordado na assistência pré-natal, sendo este o momento propício para a intervenção de profissionais de saúde (Carvalhaes, Martiniano, Malta, Takito \& Benício, 2013, Silva, Araújo, Santana, Lima, Cecchino \& Silva, 2015, Tavares, Melo, Amorim, Barros, Takito, Benício \& Cardoso, 2009). Tal intervenção deve ter o propósito de aumentar a adesão ao exercício a longo prazo, quando as gestantes devem ser estimuladas a eleger o tipo de atividade que melhor se adapte às suas características e interesses (Nascimento et al., 2014).

Diante disso, o objetivo dessa revisão integrativa foi identificar os determinantes da não adesão à prática de exercício físico durante a gestação, para que os profissionais de saúde envolvidos na assistência pré-natal possam identificar e intervir na atenuação desses fatores limitantes.

\section{Metodologia}

Trata-se de uma revisão integrativa elaborada em seis etapas: identificação da questão de pesquisa; estabelecimento de critérios de inclusão e exclusão de estudos/amostragem ou busca da literatura; definição das informações a serem extraídas dos estudos selecionados e categorização dos estudos; avaliação dos estudos incluídos na revisão integrativa; interpretação dos resultados; e apresentação da revisão/síntese do conhecimento (Mendes, Silveira \& Galvão, 2008).

A questão norteadora dessa pesquisa foi: Quais os determinantes da não adesão da prática de exercício físico durante a gestação? As buscas foram realizadas entre os meses de março a agosto de 2017, nas bases de dados Scientific Electronic Library Online (SciELO), Medical Literature Analysis and Retrieval System Online (MEDLINE), Literatura Latino-Americana e do Caribe em Ciências da Saúde (LILACS), Cochrane Library e Science Direct. Utilizou-se os descritores, definidos a partir dos termos indexados no vocabulário estruturado DeCS (Descritores em Ciências da Saúde), em português e inglês: "exercício" (exercise), "gestante"(pregnant women), "motivação" (motivation), "saúde materno-infantil" (maternal and child health), "cuidado pré-natal" (prenatal care) e "adesão ao tratamento" (patient compliance). Foi utilizado o operador booleano "and" para sistematizar as buscas, fornecendo a intercessão, uma vez que este mostra somente artigos que contenham todos os descritores digitados, restringindo a amplitude da pesquisa.

Para a inclusão dos artigos localizados nessa busca, foram adotados os seguintes critérios: 1) publicado entre janeiro de 2006 e dezembro de 2016,2) publicado em idiomas inglês, espanhol ou português, 3) disponíveis na íntegra on line e 4) que evidenciaram os determinantes da não adesão de gestantes com risco habitual à prática de exercício físico. Foram excluídos: 1) os artigos repetidos, 2) artigos teóricos (revisão de literatura, metanálise ou diretrizes) , e 3) que não apresentaram temática compatível com os descritores empregados nas buscas. 
$\mathrm{Na}$ sequência, os artigos que foram incluídos na revisão integrativa foram analisados através do Atlas Ti: The Qualitative Data Analysis \& Research Software. Todos os artigos utilizados na revisão integrativa foram migrados para o software onde foram analisados e codificados com etiquetas que remetiam aos determinantes da não adesão à prática de exercício físico durante a gestação.

\section{Resultados E Discussão}

A busca eletrônica em bases de dados identificou-se inicialmente 681 artigos. A partir da leitura do título realizou-se o primeiro refinamento da pesquisa, no qual excluiu-se 488 artigos. Na fase seguinte, de leitura dos resumos, foram eliminados 167 artigos. Desse modo, os 77 artigos oriundos das fases de leitura dos títulos e dos resumos, foram analisados e levados para consenso entre os pesquisadores, processo o qual selecionou 56 estudos para serem incluídos na análise da revisão integrativa.

Grande parte dos artigos excluídos se deu pela utilização do termo patient compliance, que remete à cooperação voluntária do paciente ao seguimento do regime de prescrição. Contudo, através da leitura dos resumos, era notório que o termo remetia a outros tipos de tratamento, como por exemplo, de tabagismo e de diabetes gestacional (sem a participação do exercício físico como tratamento não medicamentoso). Desta forma, constatou-se que o modelo biomédico ainda é um limitante da recomendação à prática de exercícios físicos para gestantes na consulta de pré-natal.

\section{Caracterização dos estudos selecionados}

Quanto ao ano de publicação, 19,65\% (11 estudos) foram publicados no período de 2006 a 2010 e 80,35\%(45 estudos) entre os anos de 2011 a 2016. Pode- se perceber que o volume de estudos aumentou nos últimos seis anos. No que tange ao quantitativo de publicações por país, obteve-se achados que abrangeram a população de gestantes de aproximadamente 21 países, uma vez que alguns estudos apenas mencionam os que foram desenvolvidos no Reino Unido, sem especificar o país. A maioria foi realizada nos Estados Unidos (18; 32,14\%), Reino Unido (9; 16,06\%) e Canadá (5; 8,92\%). O Brasil ficou em sexto lugar, juntamente com a Dinamarca, ambos com 3,57\% (2 estudos) das publicações. Esse panorama denota a carência de estudos que abordem exercício físico durante a gestação na população brasileira.

Os motivos da não adesão à prática de atividade física, em geral, durante o período gestacional foram investigados por apenas 39,28\% (22 estudos) dos achados. O maior volume de artigos (46,42\%; 26 estudos) está voltado para estudos experimentais que tinham o objetivo de investigar: benefícios da atividade física em geral e peso da gestante $(10,71 \% ; 6)$, fatores relacionados ao exercício físico e à diabetes gestacional $(10,71 \%$; 6), efeitos do exercício físico nos desfechos perinatais $(8,90 \% ; 5)$ e estilo de vida $(5,35 \% ; 3)$.

De uma forma geral, notou-se que, nas amostras das pesquisas selecionadas para revisão, identificou-se que $2 \%$ das gestantes eram nulíparas, $1 \%$ mulheres com diabetes gestacional, $1 \%$ grávidas fisicamente inativas e $1 \%$ mulheres com idades entre 16 a 30 anos e mais de 30 semanas gestacional. Outras características observadas se referem ao: estado civil - um estudo feito com 25 gestantes solteiras; ao peso gestacional - outro com 14 mulheres obesas ou com sobrepeso; e hábitos de tabagismo - dois estudos realizados especificamente com gestantes fumantes, somando um total de 821 mulheres, $3 \%$ do total geral.

Para melhor compreensão dos dados obtidos, definiu-se os determinantes da não adesão à prática de exercício físico durante a gestação como resultantes das interações individuais com o meio que influenciam a gestante a apresentar comportamentos que contribuem para a não adesão à prática de exercício físico. Por conseguinte, os achados da revisão foram categorizados segundo o Modelo Socioecológico que agrupa os determinantes da não adesão à um determinado comportamento em: fatores intrapessoais, processos interpessoais, fatores ambientais e políticas públicas (Sallis, Owen \& Fisher, 2008). 


\section{Fatores Intrapessoais}

Abrangem os elementos relacionados intrinsecamente à gestante, incluindo as seguintes variáveis: fatores socioeconômicosfatores físicos, fatores psicológicos e fatores comportamentais, que estão definidos no Quadro 1.

Gesche, Renault, Nørgaard \& Nilas (2014), Gjestland, Bø, Owe \& Eberhard-Gran (2013), Marshall, Bland \& Melton (2013), Mudd, Nechuta, Pivarnik \& Paneth (2009), Santos, et al. (2014), Yeo, Cisewski, Lock \& Marron (2010) e Whitford \& Jones (2011) preconizam que um dos fatores que pode justificar a redução do nível de atividade física durante a gestação é a idade.

Outro fator socioeconômico considerado como variável foi o nível de escolaridade, pois gestantes com baixo nível de escolaridade tendem a ter concepções equivocadas relacionadas à prática de exercício durante a gestação, como por exemplo, sem importância (Doran \& Davis, 2011, Newhan, Allan, Leahy-Warren, Carrick-Sen \& Alderdice, 2016), desnecessária (Whitford \& Jones, 2011) e prejudicial para o feto (Atkinson, Shaw \& French, 2016, Connelly, Brown, Pligt \& Teychenne, 2015, Costa \& Ireland, 2013, Gjestland et al., 2013, Groth \& Morrison-Beedy, 2013, Haakstad, Voldner, Henriksen \& Bø, 2009, Leiferman, Swibas, Koiness, Marshall \& Dunn, 2011; Muzigaba, Kolbe-Alexander \& Wong, 2014, Marshall et al., 2013; Newhan et al., 2016; Petherick, et al., 2016; Santos et al., 2014; Yeo et al., 2010).

No que concerne à renda familiar, um estudo com 4.471 gestantes, verificaram que as mais pobres eram menos propensas a se engajarem em atividades físicas, além de outros autores obtiveram dados convergentes ao supracitado (Connelly et al., 2015, Costa \& Ireland, 2013, Dlugonski \& Motl, 2016, Goodrich, Cregger, Wilcox \& Liu, 2013, Groth \& Morrison-Beedy, 2013, Haakstad et al., 2009, Marshall et al., 2013, Newhan et al., 2016, Santos et al., 2014, Silveira \& Segre, 2012, Yeo et al., 2010, Yeo, 2009, Yeo \& Logan, 2014, Whitford, Alder \& Jones, 2007, Whitford \& Jones, 2011).

Corroborando com os achados anteriores sobre as variáveis pertinentes aos Fatores Socioeconômicos, Goodrich et al. (2013), Leiferman et al. (2011), Muzigaba et al. (2014), Thornton, Kieffer, Salabarría-Peña, Odoms-Young, Willis, Kim \& Salinas (2006) e Weir, Bush, Robson, McParlin, Rankin \& Bell (2010) afirmam que residir em locais com altos índices de violência urbana é considerada uma barreira para a prática de exercício físico durante a gestação.

A última variável encontrada no que tange aos Fatores Socioeconômicos foi a ocupação das gestantes com tarefas domiciliares e laborais. Nesse contexto, Doran e Davis (2011) encontraram que cuidar de outras pessoas foi a segunda maior barreira reportada pelas gestantes acerca da prática de exercício físico. Esses achados convergem com os resultados de outros estudos que reportam barreiras relacionadas à rotina trabalhista (cansaço físico, falta de tempo em decorrência das responsabilidades trabalhistas e ainda possuírem ocupações consideradas fisicamente ativas) (Atkinson et al., 2016, Connelly et al., 2015, Groth \& Morrison-Beedy, 2013, Haakstad et al., 2009, Leiferman et al., 2011, Marshall et al., 2013, Muzigaba et al., 2014, Petherick et al., 2016, Santos et al., 2014, Stafne, et al., 2012, Torres, et al., 2016, Ussher, et al., 2008; Yeo et al., 2010).

Vale destacar que Fatores Físicos é uma das variáveis mais relevantes, pois trata dos elementos primordiais e específicos do estado gestacional. Alguns estudos encontraram os seguintes sintomas gestacionais como barreiras para a prática de exercício físico: cansaço, náusea, desconforto físico (Mudd et al., 2009), falta de energia (Anjana, et al., 2016; Atkinson et al., 2016; Choi, Lee, Vittinghoff \& Fukuoka, 2016, Connelly et al., 2015, Costa \& Ireland, 2013, Doran \& Davis, 2011, Groth \& Morrison-Beedy, 2013, Leiferman et al., 2011, Oostdam, et al., 2012, Robledo-Colonia, Sandoval-Restrepo, Mosquero-Valderrama, Escobar-Hurtado \& Ramírez-Vélez 2012, Weir et al., 2010), dores musculares e articulares (Atkinson et al., 2016, Connelly et al., 2015, Costa \& Ireland, 2013, Doran \& Davis, 2011, Duncombe, Wertheim, Skouteris, Paxton \& Kelly, 2009, Gaston \& Prapavessis, 2014, 2013; Goodrich et al., 2013, Groth \& Morrison-Beedy, 2013, Haakstad 
et al., 2009, Huberty, Buman, Leiferman, Bushar \& Adams, 2016, Leiferman et al., 2011; Marshall et al., 2013; Stafne et al., 2012; Ussher et al., 2008).

Dentre as variáveis dos Fatores Psicológicos, motivação intrínseca apresentou o maior volume de achados. Nesse sentido, a falta de auto-disciplina foi classificada como uma das três principais barreiras para a prática de exercício físico durante a gestação (Marshall et al., 2013) junto com a autoconsciência (Torres et al., 2016, Ussher et al., 2015). Outras investigações indicam que uma motivação reduzida contribui para diminuir os níveis de atividade física em gestantes (Connelly et al., 2015, Goodrich et al., 2013, Groth \& Morrison-Beedy, 2013, Huberty et al., 2016, Leiferman et al., 2011, Marshall et al., 2013, Mullan, Henderson, Kothe, Allom, Orbell \& Hamilton, 2016, Muzigaba et al., 2014, Newhan et al., 2016, Yeo et al., 2010, Weir et al., 2010), assim como a baixa autoeficácia (Costa \& Ireland, 2013, Gaston \& Prapavessis, 2013, Groth \& MorrisonBeedy, 2013).

As duas variáveis que se destacaram nos Fatores Comportamentais foram: histórico da prática de exercício físico antes da gestação (a ausência do mesmo ou experiências negativas) e aulas individuais. Em relação à primeira, Costa \& Ireland (2013), Devlin, Huberty \& Downs (2016), Haakstad et al. (2009), Leiferman et al. (2011), Marshall et al. (2013), Mullan et al. (2016) e Thornton et al. (2006) comprovam que a ausência de um histórico de prática é fator determinante da não adesão. Ademais, Doran e Davis (2011) acrescentam que a gestante ter um histórico de prática com algum tipo de lesão também é prejudicial para a adesão.

No que tange às características peculiares do tipo de prática de exercício físico, encontrou-se que grupos de mulheres grávidas que se exercitam juntos ou estabelecem um local de prática de exercício físico em suas respectivas comunidades tendem a incentivar a sua participação na prática de exercício físico durante a gravidez (Backhausen, et al., 2014, Muzigaba et al. 2014, Seneviratne, et al., 2016, Yeo \& Logan, 2014, Weir et al., 2010). 
Quadro 1. Síntese dos motivos da não adesão à prática de exercícios físicos durante a gestação relacionados aos Fatores Intrapessoais.

\begin{tabular}{|c|c|c|c|}
\hline \multicolumn{2}{|l|}{ Variäweis } & putores & \multirow{2}{*}{$\begin{array}{l}\text { Frequencia } \\
\text { ele estuclos } \\
14,3 \% \\
0=8\end{array}$} \\
\hline $\begin{array}{l}\text { Fatores } \\
\text { Sacioueconomicas }\end{array}$ & 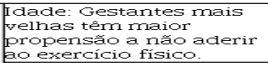 & 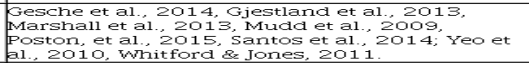 & \\
\hline & 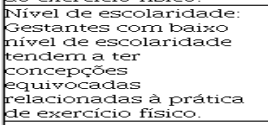 & 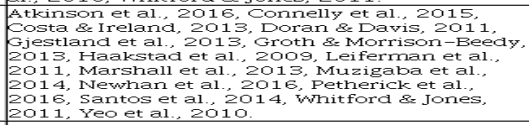 & $n=15$ \\
\hline & 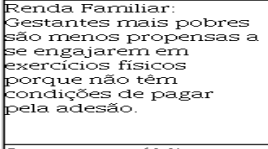 & 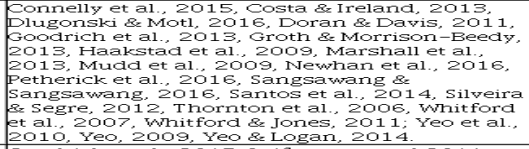 & $m=2 \infty, 7 \%$ \\
\hline & 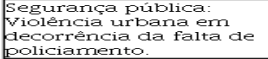 & 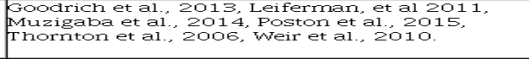 & $\begin{array}{l}10,7 \% \\
(n=6 \%\end{array}$ \\
\hline & 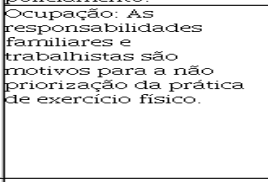 & 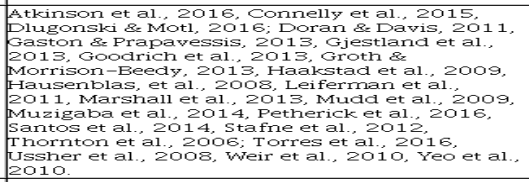 & $(n=22396$ \\
\hline \multirow[t]{3}{*}{ Fatores Fisicos } & 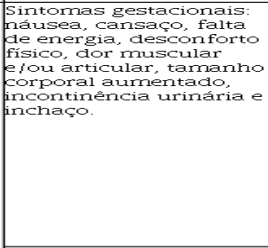 & 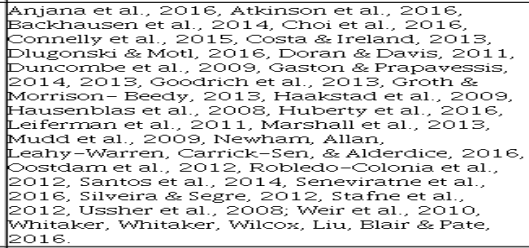 & $\begin{array}{r}51,896 \\
(n=\geq 99\end{array}$ \\
\hline & 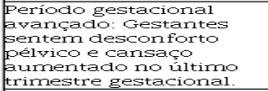 & $\begin{array}{l}\text { Leiferman et al, } 2011 \text {, Mudd et al } 2009 \text {, } \\
\text { Senerirattre et al., 2016, Yeo, Zaos. }\end{array}$ & $7.1 \%(n=4)$ \\
\hline & 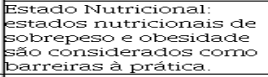 & 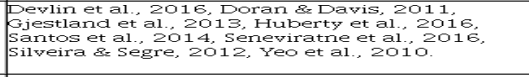 & $14,3 \%(\mathrm{n}=8)$ \\
\hline \multirow[t]{7}{*}{$\begin{array}{l}\text { Fatores } \\
\text { Psicologicos }\end{array}$} & $\begin{array}{l}\text { Problemas psicológicos: } \\
\text { fomb ansiedaace e } \\
\text { listurbias de } \\
\text { hutoirnagem. }\end{array}$ & $\begin{array}{l}\text { Devlin et al., } 2016 \text {, Haakstad et al., } 2009 \text {, } \\
\text { Leifermian et al, za11 }\end{array}$ & $5,3 \%(n=3)$ \\
\hline & 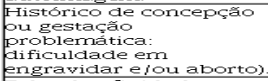 & $\begin{array}{l}\text { Atkinson et al.. } 2016 \text {. Devlin et al., } 2016 . \\
\text { Huberty et al., } 2016 .\end{array}$ & $5.3 \%(n=3)$ \\
\hline & $\begin{array}{l}\text { Falta de afinidade com } \\
\text { pratica: Naogosta de } \\
\text { praticar exercicio fisich. }\end{array}$ & $\begin{array}{l}\text { Connely et al. } 2015 \text {, Doran a Davis, } 2011 \\
\text { Euncombe et al, 2009, Whitford } \mathrm{z} \text { Jones, } 2011\end{array}$ & $8.9 \%(n=5)$ \\
\hline & 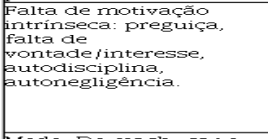 & 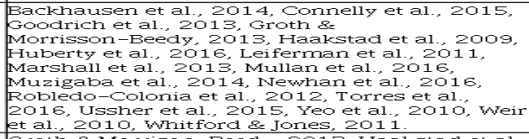 & $\begin{array}{l}30,3 \% 6 \\
\infty=17\end{array}$ \\
\hline & $\begin{array}{l}\text { Medo: De machucar o } \\
\text { Mebe ou prejudicaro } \\
\text { desenvolvimento do } \\
\text { feto. }\end{array}$ & 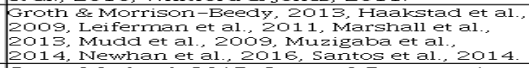 & $\begin{array}{c}14,3 \% \\
61-8 \%\end{array}$ \\
\hline & $\begin{array}{l}\text { Falta de autce ficácia: } \\
\text { incapacidaded } \\
\text { inabilicade de praticar } \\
\text { exercicio fisico. }\end{array}$ & 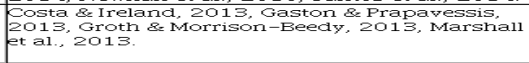 & $7.196(n=4)$ \\
\hline & 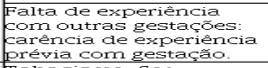 & 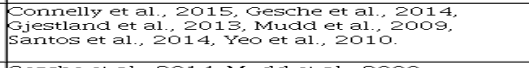 & $(\mathrm{I}=\mathrm{E})$ \\
\hline \multirow{8}{*}{$\begin{array}{l}\text { Fatores } \\
\text { Eomportamentais } \\
\text { Fatores } \\
\text { Eomportamentais }\end{array}$} & $\begin{array}{l}\text { Tabazismo: ser } \\
\text { furnante. }\end{array}$ & Gesche et al., 2014, Mudd et al., 2009. & $3,6 \%(n=2)$ \\
\hline & 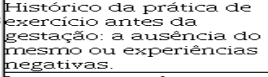 & 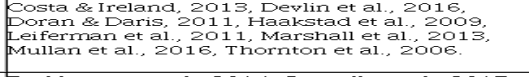 & $14,396$ (II -8$)$ \\
\hline & $\begin{array}{l}\text { Msegurança: aurante a } \\
\text { exsecuça do exercicin. }\end{array}$ & 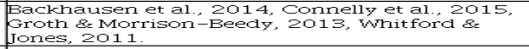 & $7.1 \%(n=4)$ \\
\hline & 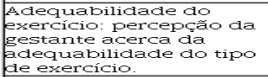 & Atkinson et al. 2016 , Yeo, 2009 . & $3.6 \%(n=2)$ \\
\hline & 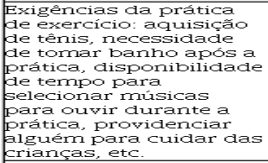 & $\begin{array}{l}\text { Atkinson et al., } 2016 \text {. Seneviratne et al., } 2016 \text {. } \\
\text { yeo, 2000. }\end{array}$ & $5.3 \%(n-3)$ \\
\hline & $\begin{array}{l}\text { Característica da } \\
\text { Orática: aulas } \\
\text { individuais sao } \\
\text { desmotivantes. }\end{array}$ & 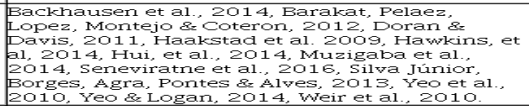 & $\begin{array}{l}21,4 \% \\
(1)=1 \geq 2\end{array}$ \\
\hline & 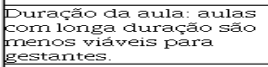 & Sangsawang \& sarngsawarig, 201 & $1,8 \%(\mathbf{n}=\mathbf{1})$ \\
\hline & 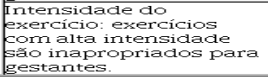 & freo, 2000 & $1.8 \%(n=1)$ \\
\hline
\end{tabular}




\section{Processos Interpessoais}

Refere-se aos processos relacionais construídos com cônjuge, familiares, amigos, profissionais de saúde e sociedade com os quais a gestante convive, foi educada e interage cotidianamente. As variáveis encontradas foram divididas em: suporte familiar, suporte social e suporte assistencial, que estão definidos no Quadro 2.

A primeira variável remete aos familiares que criticam a gestante no que concerne à prática de exercício físico durante a gestação. Atkinson et al. (2016) realizaram uma investigação na Inglaterra e encontraram que gestantes que optaram por adotar um estilo de vida fisicamente ativo foram criticadas pelos seus familiares, o que gerou uma influência negativa e equivocada. Em contrapartida, gestantes latinas residentes nos Estados Unidos relataram que seus maridos constituíram a fonte mais importante de suporte informacional para o aumento da atividade física. Eles sempre disseram às suas esposas para se exercitarem e perderem peso na gravidez para que elas não se tornassem obesas ou doentes e este conselho geralmente resultou em mulheres envolvidas em mais atividades físicas (Thornton et al., 2006).

A maioria dos achados menciona a importância do suporte dado pelos amigos das gestantes, tanto no que se refere ao apoio em tarefas cotidianas para que a gestante tenha tempo para praticar exercício físico, como encorajamento verbal, companhia durante a prática e até mesmo amigas que servem/serviram como exemplos de gestantes que tinham estilo de vida fisicamente ativo (Atkinson et al., 2016, Connelly et al., 2015, Dlugonski \& Motl, 2016, Doran \& Davis, 2011, Groth \& Morrison-Beedy, 2013, Hawkins et al., 2014, Huberty et al., 2016, Leiferman et al., 2011, Marshall et al., 2013, Oostdam et al., 2012, Petherick et al., 2016, Thornton et al., 2006, Whitaker et al., 2016).

As variáveis encontradas foram referentes à proibição médica da prática de exercício, mesmo a gestante sendo diagnosticada como saudável, o que denota a necessidade de capacitação multidisciplinar dos profissionais atuantes na assistência pré-natal (Connelly et al., 2015). No mesmo âmbito, vários autores reiteram que gestantes saudáveis e fisicamente inativas nunca receberam nenhum profissional da saúde que lhes deu assistência pré-natal ou sequer mencionou o termo exercício físico (Connelly et al., 2015, Costa \& Ireland, 2013, Doran \& Davis, 2011, Goodrich et al., 2013, Groth \& Morrison-Beedy, 2013, Haakstad et al., 2009, Leiferman et al., 2011, Lindqvist, Lindqvist, Eurenius, Persson, Ivarsson \& Mogren, 2016, Muzigaba et al., 2014, Shirazian, Monteith, Friedman \& Rebarber, 2010, Yeo \& Logan, 2014, Weir et al., 2010, Whitaker et al., 2016).

No que tange à carência de intervenções em saúde que possam aproximar o profissional de saúde da gestante, os achados mencionam que essas ações: aprimoram os cuidados em saúde, proporcionam maior controle dos hábitos das gestantes e emitem informações consistentes sobre a prática de exercícios físicos de forma segura (Backhausen et al., 2014, Connelly et al., 2015, Doran \& Davis, 2011, Goodrich et al., 2013, Hawkins et al., 2014, Hui et al., 2014, Leiferman et al., 2011, Marshall et al., 2013, Mudd et al., 2009, Muzigaba et al., 2014, Paul \& Olson, 2013, Pelaez, Gonzalez-Cerron, Montejo \& Barakat, 2014, Ussher et al., 2015, Weir et al., 2010, Whitford \& Jones, 2011).

Por fim, no estudo de Backhausen et al. (2014), foi salientada a importância do acompanhamento do profissional de Educação Física desde a prescrição até o acompanhamento da execução dos exercícios físicos, garantindo a segurança da prática. 
Quadro 2. Síntese dos motivos da não adesão à prática de exercícios físicos durante a gestação relacionados aos Processo Interpessoais.

\begin{tabular}{|c|c|c|c|}
\hline \multicolumn{2}{|l|}{ Variáveis } & \multirow{2}{*}{$\begin{array}{l}\text { Autores } \\
\text { Atkinson et al., 2016, Gaston \& Prapavessis, } \\
\text { 2013, Haakstad et al., 2009, Leiferman et al., } \\
\text { 2011, Thornton et al., 2006, Whitaker et al., } \\
2016\end{array}$} & \multirow{2}{*}{$\begin{array}{l}\text { Frequência } \\
\text { de estudos } \\
10,7 \% \\
(n=6)\end{array}$} \\
\hline \begin{tabular}{|l} 
Suporte \\
Familiar
\end{tabular} & $\begin{array}{l}\text { Críticas familiares: } \\
\text { criticismo por parte dos } \\
\text { familiares acerca do estilo } \\
\text { de vida ativo da gestante. }\end{array}$ & & \\
\hline & $\begin{array}{l}\text { Carência de } \\
\text { incentivo /apoio da } \\
\text { família: ausência de } \\
\text { encorajamento e apoio por } \\
\text { parte do conjuge e da } \\
\text { familia. }\end{array}$ & $\begin{array}{l}\text { Atkinson et al., 2016, Costa \& Ireland, 2013, } \\
\text { Plugonski \& Motl, 2016, Doran \& Davis, 2011, } \\
\text { Gesche et al., 2014, Gjestland et al., 2013, } \\
\text { Goodrich et al., 2013, Groth \& } \\
\text { Morrison-Beedy, 2013, Leiferman et al., 2011, } \\
\text { Mudd et al., 2009, Muzigaba et al., 2014, } \\
\text { Newhan et al., 2016, Petherick et al., 2016, } \\
\text { Santos et al., 2014, Thornton et al., 2006, Weir } \\
\text { et al., 2010, Whitaker et al., } 2016\end{array}$ & $\begin{array}{c}28,6 \% \\
(\mathrm{n}=16)\end{array}$ \\
\hline \multirow[t]{4}{*}{$\begin{array}{l}\text { Suporte } \\
\text { Social }\end{array}$} & $\begin{array}{l}\text { Carência de incentivo dos } \\
\text { amigos: ausência de } \\
\text { encorajamento por parte } \\
\text { dos amigos. }\end{array}$ & $\begin{array}{l}\text { Atkinson et al., 2016, Connelly et al., 2015, } \\
\text { Dlugonski \& Motl, 2016, Doran \& Davis, } 2011 \text {, } \\
\text { Groth \& Morrison-Beedy, 2013, Hawkins et al., } \\
\text { 2014, Huberty et al., 2016, Leiferman et al., } \\
\text { 2011, Oostdam et al., 2012, Petherick et al., } \\
\text { 2016, Thornton et al., 2006, Whitaker et al., } \\
\text { 2016. }\end{array}$ & $\begin{array}{c}21,4 \% \\
(n=12)\end{array}$ \\
\hline & $\begin{array}{l}\text { Carência de apoio nas } \\
\text { atividades diárias: } \\
\text { ausência de alguém que } \\
\text { cuide das crianças } \\
\text { enquanto a gestante } \\
\text { pratica exercício físico. } \\
\end{array}$ & Dlugonski \& Motl, 2016. & $1,8 \%(n=1)$ \\
\hline & $\begin{array}{l}\text { Opiniốes equivocadas: } \\
\text { Convivio com pessoas que } \\
\text { tenham crenças } \\
\text { equivocadas acerca da } \\
\text { prática de exercício físico } \\
\text { durante a gestação. }\end{array}$ & $\begin{array}{l}\text { Atkinson et al., 2016, Marshall et al., 2013, } \\
\text { Thornton et al., 2006, Whitaker et al., } 2016 \text {. }\end{array}$ & $7,1 \%(n=4)$ \\
\hline & $\begin{array}{l}\text { Mídia: informações } \\
\text { transmitidas pela midia. }\end{array}$ & Atkinson et al., 2016 & $1,8 \%(n=1)$ \\
\hline \multirow[t]{4}{*}{$\begin{array}{l}\text { Suporte } \\
\text { Assistencial }\end{array}$} & $\begin{array}{l}\text { Proibição médica: receber } \\
\text { proibição médica para a } \\
\text { prática de exercício físico } \\
\text { mesmo sem apresentar } \\
\text { complicações gestacionais. }\end{array}$ & Connelly et al., 2015. & $1,8 \%(n=1)$ \\
\hline & $\begin{array}{l}\text { Falta de informaçãol } \\
\text { recommendação: Nấo } \\
\text { receber informação e nem } \\
\text { recomendação acerca da } \\
\text { prática de exercício físico } \\
\text { durante a gestação. }\end{array}$ & $\begin{array}{l}\text { Connelly et al., 2015, Costa \& Ireland, 2013, } \\
\text { Poran \& Davis, 2011, Goodrich et al., 2013, } \\
\text { Groth \& Morrison- Beedy, 2013, Haakstad et } \\
\text { al., 2009, Leiferman et al., 2011, Lindqvist et } \\
\text { al., 2016, Muzigaba et al., 2014, Shirazian et } \\
\text { al., 2010, Yeo \& Logan, 2014, Weir et al., } \\
\text { 2010, Whitaker et al., 2016. }\end{array}$ & $\begin{array}{l}23,2 \% \\
(n=13)\end{array}$ \\
\hline & $\begin{array}{l}\text { Carência de } \\
\text { acompanhamento } \\
\text { profissional: Não ter } \\
\text { acompanhamento do } \\
\text { profissional de Educação } \\
\text { Física durante a prática de } \\
\text { exercicio físico. }\end{array}$ & Backhausen et al., 2014, Hawkins et al., 2014. & $3,6 \%(n=2)$ \\
\hline & $\begin{array}{l}\text { Carência de intervenções: } \\
\text { voltadas para a prática de } \\
\text { exercício físico durante a } \\
\text { gestaçấo promovidas por } \\
\text { profissionais da saúde. }\end{array}$ & $\begin{array}{l}\text { Backhausen et al., 2014, Connelly et al., 2015, } \\
\text { Poran \& Davis, 2011, Goodrich et al., 2013, } \\
\text { Hawkins et al., 2014, Hui et al., 2014, } \\
\text { Leiferman et al., 2011, Marshall et al., 2013, } \\
\text { Mudd et al., 2009, Muzigaba et al., 2014, Paul } \\
\& \text { Olson, 2013, Pelaez et al., 2014, Ussher et } \\
\text { al., 2015, Weir et al., 2010, Whitford \& Jones, } \\
\text { 2011. }\end{array}$ & $\begin{array}{c}26,8 \% \\
(n=15)\end{array}$ \\
\hline
\end{tabular}




\section{Fatores Ambientais}

É um agrupamento referente ao ambiente físico no qual a gestante vive e com o qual interage. Isso inclui os fatores ambientais naturais, como clima ou ambiente construído, assim como a configuração física das cidades e da vizinhança, os serviços oferecidos pelo comércio local e o ambiente de trabalho. As variáveis foram classificadas em: condições meteorológicasferta de serviços e ambiente de prática, que estão definidos no Quadro 3.

A variável das Condições Meteorológicas remete às mudanças climáticas que foram caracterizadas pelos autores como barreiras ambientais (Hui et al., 2014). Gestantes latinas residentes no México e nos EUA afirmaram que as barreiras ambientais interferem nas questões de locomoção e acesso aos locais de prática (Leiferman et al., 2011).

A falta de oferta de serviços voltados à prática de exercícios físicos, como por exemplo: academias, próximos à residência da gestante foi uma barreira relatada pelos autores que vai desde problemas de disponibilidade de espaços privados quanto públicos (Haakstad et al., 2009, Muzigaba et al., 2014, Osuji, Lovegreen, Elliot \& Brownson, 2006).

Os estudos de Barakat et al. (2012) e Barakat, Cordero, Coteron, Luaces \& Montejo (2011) preconizam que a temperatura adequada de um ambiente é fator relevante por conta da temperatura corporal da gestante que deve evitar temperaturas acima de $32^{\circ} \mathrm{C}$. Os mesmos autores acrescentam que um ambiente com música agradável também pode ser fator motivante para a prática. Marshall et al. (2013) desenvolveram um estudo com gestantes norte-americanas residentes de uma comunidade rural e encontram achados semelhantes.

Quadro 3. Síntese dos motivos da não adesão à prática de exercícios

físicos durante a gestação relacionados aos Fatores Ambientais.

\begin{tabular}{|c|c|c|c|}
\hline \multicolumn{2}{|l|}{ Variáveis } & \multirow{2}{*}{\begin{tabular}{|l} 
Autores \\
Hui et al., 2014, Leiferman et al., \\
2011
\end{tabular}} & \multirow{2}{*}{\begin{tabular}{|l}
$\begin{array}{l}\text { Frequência } \\
\text { de estudos }\end{array}$ \\
$3,6 \%(n=2)$
\end{tabular}} \\
\hline \begin{tabular}{|l|} 
Condições \\
Metereológicas
\end{tabular} & $\begin{array}{l}\text { Condições meteorológicas: não são } \\
\text { favoráveis à prática de exercício } \\
\text { físico, assim como não } \\
\text { permitem/dificultam o acesso aos } \\
\text { serviços que a promovem. }\end{array}$ & & \\
\hline $\begin{array}{l}\text { Oferta de } \\
\text { serviços }\end{array}$ & $\begin{array}{l}\text { Carência de oferta de serviços: } \\
\text { nâo existem serviços voltados à } \\
\text { prática de exercícios físicos, como } \\
\text { por exemplo: academias, próximos } \\
\text { à residência da gestante. }\end{array}$ & $\begin{array}{l}\text { Haakstad et al., 2009, Muzigaba et } \\
\text { al., 2014, Osuji et al., 2006. }\end{array}$ & $5,3 \%(n=3)$ \\
\hline $\begin{array}{l}\text { Ambiente de } \\
\text { prática } \\
\text { assistencial }\end{array}$ & $\begin{array}{l}\text { Inadequabilidade do ambiente: No } \\
\text { ambiente de prática de exercício } \\
\text { físico a temperatura não é } \\
\text { adequada para gestantes } \\
\text { (temperatura }=19-21^{\circ} \mathrm{C} \text {; } \\
\text { (humidade }=50-60 \% \text { ). }\end{array}$ & $\begin{array}{l}\text { Barakat et al., 2012, Barakat et al., } \\
\text { 2011, Marshall et al., 2013, Osuji } \\
\text { et al., } 2006\end{array}$ & $7,1 \%(n=4)$ \\
\hline
\end{tabular}

\section{Políticas Públicas}

Engloba fatores políticos que influenciam a vida cívica da gestante a nível internacional, nacional, regional e individual. As variáveis encontradas foram divididas em: transporte público, autoridades locais e publicidade, que estão definidos no Quadro 4. 
No Transporte Público foi encontrada apenas uma variável referente à inexistência de transporte público. O estudo de Costa e Ireland (2013), realizado no Canadá com 84 gestantes, e o de Oostdam et al. (2012), desenvolvido na Holanda com 101 gestantes, encontraram que a carência de transporte público acessível é uma barreira frente à prática de exercício físico.

Os estudos de Leiferman et al. (2011) e de Torres et al. (2016) também encontraram resultados semelhantes. Entretanto, merecem destaque, pois o primeiro foi realizado com base em um modelo socioecológico, com 25 gestantes norte- americanas de baixo nível socioeconômico e utilizou o software Atlas $T i$ para fazer análise e codificação das entrevistas realizadas. O segundo foi realizado em Porto Rico com 200 gestantes hispânicas de comunidades com barreiras econômicas, ambientais, culturais e sociais.

Na subdimensão Autoridades Locais encontrou-se duas variáveis: não existência de programa/serviços de prática de exercício físico para gestantes que tenham baixo custo e flexibilidade de opções de exercícios (à domicílio ou em grupo) e a inexistência de programas ou equipamentos públicos para a prática de exercício físico para gestantes com acompanhamento profissional no bairro onde moram.

Os estudos que deram embasamento teórico para esses achados foram realizados nos EUA, Canadá, África do Sul e Escócia. Vale ressaltar a necessidade de investimento por parte do poder público no que concerne à criação de programas/serviços gratuitos com a presença do profissional de Educação Física, assim como a realização de parcerias público-privadas com o intuito de viabilizar o acesso de gestantes com baixa renda a serviços que forneçam a prática de exercícios físicos (Doran \& Davis, 2011, Goodrich et al., 2013, Leiferman et al., 2011, Marshall et al., 2013, Mudd et al., 2009, Muzigaba et al. 2014, Torres et al., 2016, Yeo \& Logan, 2014, Whitaker et al., 2016, Whitford \& Jones, 2011, Wong, 2014).

Apenas quatro achados apontam para a importância da Publicidade - distribuição de materiais informativos acerca da prática de exercícios físicos durante a gestação. Observou-se que três destes foram desenvolvidos no continente europeu com gestantes saudáveis e obesas/em sobrepeso. Na apresentação dos resultados da validação do instrumento será questionada a relevância da inclusão do item referente a essa operacionalização, a baixa importância dada ao item pode ser justificada pela cultura brasileira de não desenvolver/valorizar esse meio de comunicação entre o sistema de saúde e a população (Haakstad et al., 2009, Leiferman et al., 2011, Oostdam et al., 2012, Weir et al., 2010). 
Quadro 4. Síntese dos motivos da não adesão à prática de exercícios físicos durante a gestação relacionados às Políticas Públicas.

\begin{tabular}{|c|c|c|c|}
\hline \multicolumn{2}{|l|}{ Variáveis } & \multirow{2}{*}{$\begin{array}{l}\text { Autores } \\
\text { Leiferman et al., 2011, Ussher et al., } \\
2015 .\end{array}$} & \multirow{2}{*}{$\begin{array}{l}\text { Frequência } \\
\text { de estudos }\end{array}$} \\
\hline \begin{tabular}{|l|} 
Transporte \\
público
\end{tabular} & $\begin{array}{l}\text { Carência de transporte público: não } \\
\text { existe transporte público (ônibus, } \\
\text { trem, metrô) que facilite o acesso } \\
\text { aos locais que oferecem serviços } \\
\text { voltados para a prática de exercício } \\
\text { físico para gestantes. }\end{array}$ & & \\
\hline \multirow[t]{2}{*}{$\begin{array}{l}\text { Autoridades } \\
\text { locais }\end{array}$} & $\begin{array}{l}\text { Dificuldade de acesso à prática: não } \\
\text { existem programas ou serviços de } \\
\text { prática de exercício físico para } \\
\text { gestantes com baixo custo e } \\
\text { flexibilidade de opçóes de exercícios } \\
\text { (à domicílio ou em grupo). }\end{array}$ & $\begin{array}{l}\text { Costa \& Ireland, 2013, Oostdam et } \\
\text { al., } 2012 \text {. }\end{array}$ & $3,6 \%(n=2)$ \\
\hline & $\begin{array}{l}\text { Escassez de serviços públicos: não } \\
\text { existem programas ou } \\
\text { equipamentos públicos para a } \\
\text { prática de exercício físico para } \\
\text { gestantes com acompanhamento } \\
\text { profissional no bairro onde moram. }\end{array}$ & $\begin{array}{l}\text { Doran \& Davis, 2011, Goodrich et } \\
\text { al., 2013, Leiferman et al., } 2011 \text {, } \\
\text { Marshall et al., 2013, Mudd et al., } \\
\text { 2009, Muzigaba et al., 2014, Torres } \\
\text { et al., 2016, Whitaker et al., 2016, } \\
\text { Whitford \& Jones, 2011, Yeo \& } \\
\text { Logan, 2014. }\end{array}$ & $\begin{array}{c}17,8 \% \\
(n=10)\end{array}$ \\
\hline Publicidade & $\begin{array}{l}\text { Falta de publicidade: o governo não } \\
\text { fornece panfletos ou cartilhas sobre } \\
\text { os benefícios da prática de exercício } \\
\text { físico durante a gestação. }\end{array}$ & $\begin{array}{l}\text { Haakstad et al., } 2009 \text {, Leiferman et } \\
\text { al., 2011, Oostdam et al., } 2012 \text {, } \\
\text { Weir et al., 2010. }\end{array}$ & $7,1 \%(n=4)$ \\
\hline
\end{tabular}

\section{CONCLUSÃo}

Este trabalho apresentou os motivos da não adesão à prática de exercício físico durante a gestação de risco habitual, os quais foram classificados em fatores intrapessoais, processos interpessoais, fatores ambientais e políticas públicas, para melhor compreensão das conjunturas que permeiam o cenário de inatividade física durante a gestação. Os fatores intrapessoais e os processos interpessoais apresentaram os maiores números de variáveis e as maiores frequências, 191 e 71 evidências, respectivamente.

Esse fato indica que o maior número de barreiras concerne a elementos relacionados intrinsicamente à gestante e às relações construídas com conjuge, familiares, amigos, profissionais de saúde e sociedade com os quais a gestante convive, foi educada e interage cotidianamente.

A diversidade de barreiras apresenta-se como um desafio para todos os profissionais de saúde que atuam na assistência materno-infantil.

Desta feita, salienta-se a importância da disseminação dessas evidências com as gestantes e, principalmente, com todos os profissionais da saúde que atuam na assistência materno-infantil e com os pesquisadores dessa área de conhecimento. É necessário que as gestantes reconheçam os motivos que as levam a não aderir à prática de exercício físico durante a gestação e, consequentemente, as privam da obtenção dos benefícios da prática para si e para o feto em desenvolvimento. Os profissionais da saúde terão elementos norteadores para: a qualificação profissional acerca da importância da recomendação da prática de exercício físico durante a gestação nas consultas de pré-natal e a identificação das possíveis barreiras à prática de exerício físico durante a gestação.

Ademais, urge o desenvolvimento de estudos que abordem a prática de exercício físico durante a gestação, pois apesar do aumento do volume de publicações ainda é notória a carência de estudos experimentais. 


\section{REFERENCIAS}

American College of Obstretricians and Gynecologists. (2002). Committee on Obstetric. Exercise during pregnancy and the post-partum period. Practice Bulletin n. 267. Obstretrics and Gynecology, 99, 171-173.

Anjana, R. M., Sudha V., Lakshmipriya, N., Anitha, C., Unnikrishnan, R., Bhavadharini, B., ... Mohan, V. (2016). Physical activity patterns and gestational diabetes outcomes: the wings project. Diabetes Research and Clinical Practice, 116, 253-262.

Atkinson, L., Shaw, R. L., \& French, D. P. (2016). Is pregnancy a teachable moment for diet and physical activity behavior change? An interpretative phenomenological analysis of the experiences of women during their first pregnancy. British Journal of Health Psychology, 21(4), 842-858.

Backhausen, M. G., Katballe, M., Hansson, H., Tabor, A., Damm, P. \& Hanne, K. H. (2014). A standardized individual unsupervised water exercise intervention for healthy pregnant women. A qualitative feasibility study. Sexual and Reproductive Healthcare, 5(4), 176-181.

Barakat, R., Pelaez, M., Lopez, C., Montejo, R., \& Coteron J. (2012). Exercise during pregnancy improves maternal glucose screen at 24-28 weeks: a randomised controlled trial. British Journal of The Journal of Reproductive Medicine, 46(9), 656- 661.

Barakat, R., Cordero, Y., Coteron, J., Luaces, M., \& Montejo, R. (2011). Exercise during pregnancy reduces the rate of cesarean and instrumental deliveries: results of a randomized controlled trial. The Journal of Maternal-Fetal and Neonatal Medicine, 25(11), 2372-2376.

Brasil. Ministério da Saúde. Secretaria de Atenção à Saúde. Departamento de Ações Programáticas Estratégicas. (2006). Pré-natal epuerpério: atenção qualificada e humanizada: manual técnico. Brasília: Ministério da Saúde.

Carvalhaes, M. A.B. L., Martiniano, A. C. A., Malta, M. B., Takito, M. Y., \& Benício, M. H. D. A. (2013). Atividade física em gestantes assistidas na atenção primária à saúde. Revista de Saúde Pública, 47(5), 958-967.

Caspersen, C. J., Powell, K. F., \& Christenson, G. M. (1985). Physical activity, exercise and physical fitness: definitions and distinctions for health-related research. Public Health Reports, 100, 126-131.

Choi, J., Lee, J. H., Vittinghoff, E., \& Fukuoka, Y. (2016). Health physical activity intervention: a randomized pilot study in physically inactive pregnant women. Maternal and Child Health Journal, 20(5), 1091-1101.

Connelly, M., Brown, H., Pligt, P. V. D., \& Teychenne, M. (2015). Modifiable barriers to leisure-time physical activity during pregnancy: a qualitative study investigating first time mother's views and experiences. BMC Pregnancy Childbirth, 15(1), 100.

Costa, D., \& Ireland, K. (2013). Perceived benefits and barriers to leisure-time physical activity during pregnancy in previously inactive and active women. Women Health, 53(2), 185-202.

Dempsey, J. C, Butler, C. L., \& Williams, M. A. (2004). No need for a pregnant pause: physical activity may reduce the occurrence of gestational diabetes mellitus and preeclampsya. Exercise and Sport Sciences Reviews, 33(3), 141-149.

Devlin, C. A., Huberty, J., \& Downs, D. S. (2016). Influences of prior miscarriage and weight status on perinatal psychological well-being, exercise motivation and behavior. Midwifery, 43, 29-36.

Dlugonski, D. \& Motl, R. W. (2016). Physical activity experiences and beliefs among single mothers: a qualitative study. Research Quarterly for Exercise and Sport, 87(3), 311-317.

Doran, F., \& Davis, K. (2011). Factors that influence physical activity for pregnant and postpartum women and implications for primary care. Australian Journal of Primary Health, 17(1), 79-85.

Duncombe, D., Wertheim, E. H., Skouteris, H., Paxton, S. J., \& Kelly, L. (2009). Factors related to exercise over the course of pregnancy including women's beliefs about the safety of exercise during pregnancy. Midwifery, 25(4), 430-438.

Fell, D. B., Joseph, K. S., Armson, B. A., \& Dodds, L. (2008). The impact of pregnancy on physical activity level. Maternal and Child Health Journal, 13(5), 597-603.

Garshasbi, A., \& Faghih Zadeh, S. (2005). The effect of exercise on the exercise on the intensity of low back pain in pregnant women. International Journal of Gynaecology and Obstetrics, 88(3), 271-275. 
Gaston, A., \& Prapavessis, H. (2013). Tired, moody and pregnant? Exercise may be the answer. Psychology and Health, 28(12), 1353-1369.

Gaston, A., \& Prapavessis, H. (2014). Using a combined protection motivation theory and health action process approach intervention to promote exercise during pregnancy.Journal of Behavioral Medicine, 37(2), 173-184.

Gesche, J., Renault, K., Nørgaard, K., \& Nilas, L. (2014). Representativeness of participants in a lifestyle intervention study in obese pregnant women-the difference between study participants and nonparticipants. Obesity Facts, 7(6), 351-360.

Gjestland, K., Bø, K., Owe, K. M., \& Eberhard-Gran, M. (2013). Do pregnant women follow exercise guidelines? Prevalence data among 3482 women, and prediction of low-back pain, pelvic girdle pain and depression. British Journal of Sports Medicine, 7(8), 515-520.

Goodrich, K., Cregger, M., Wilcox, S., \& Liu, J. (2013). A qualitative study of factors affecting pregnancy weight gain in African American women. Maternal and Child Health Journal, 17(3), 432- 440.

Goodwin, A., Astbury, J. \& Mcmeeken, J. (2000). Body image and psychological wellbeing in pregnancy: a comparision of exercisers and non-exercisers. Australian and New Zealand Journal of Obstetrics and Gynaecology, 40(4), $442-447$.

Groth, S. W. \& Morrison-Beedy, D. (2013). Low-income, pregnant, African American women's views on physical activity and diet. Journal of Midwifery Womens Health, 58(2), 195-202.

Haakstad, L. A. H., Voldner, N., Henriksen, T., \& Bø, K. (2009). Why do pregnant women stop exercising in the third trimester? Acta Obstetricia et Gynecologica Scandinavica, 88(11), 1267- 1275.

Hausenblas, H. A., Brewer, B. W., Van Raalte, J. L., Cook, B., Downs, D. S., Weis, C. A., Nigg, C., \& Cruz, A. (2008). Development and evaluation of a multimedia CD-ROM for exercise during pregnancy and postpartum. Patient Education and Counseling, 70(2), 215-219.

Hawkins, M., Hosker, M., Marcus, B. H., Rosal, M. C., Braun, B., Stanek, E. J., Markenson, G., \& Chasan-Taber, L. (2014). A pregnancy lifestyle intervention to prevent gestational diabetes risk factors in overweight Hispanic women: a feasibility randomized controlled trial. Diabetic Medicine, 32(1), 108-115.

Huberty, J. L., Buman, M. P., Leiferman, J. A., Bushar, J., \& Adams, M. A. (2016). Trajectories of objectively-measured physical activity and sedentary time over the course of pregnancy in women self-identified as inactive. Preventive Medicine Reports, 3, 353-360.

Hui, A. L., Back, L., Ludwig, S., Gardiner, P., Sevenhuysen, G., Dean, H. J., ... Shen, G. X. (2014). Effects of lifestyle intervention on dietary intake, physical activity level, and gestational weight gain in pregnant women with different pre-pregnancy Body Mass Index in a randomized control trial. BMC Pregnancy Childbirth, 14(331), $1-9$.

Kihlstrand, M., Stenman, B., Nilsson, S., \& Axelsson, O. (1999). Water-gymnasticis reduced the intensity of back/low back pain in pregnant women. Acta Obstetricia et Gynecologica Scandinavica, 87(3), 180-185.

Leiferman, J., Swibas, T., Koiness, K., Marshall, J. A., \& Dunn, A. L. (2011). My baby, my move: examination of perceived barriers and motivating factors related to antenatal physical activity. Journal of Midwifery Womens Health, 56(1), 33-40.

Lindqvist, M., Lindkvist, M., Eurenius, E., Persson, M., Ivarsson, A., \& Mogren, I. (2016). Leisure time physical activity among pregnant women and its associations with maternal characteristics and pregnancy outcomes. Sexual and Reproductive Healthcare, 9, 14-20.

Marquez-Sterling, S., Perry, A. C., Kaplan, T. A., Halberstein, R. A., Signorile, J. F. (2000). Physical and psychological changes with vigorous exercise in sedentary primigravidae. Medicine and Science in Sports Exercise, 32(1), 58-62.

Marshall, E. S., Bland, H., \& Melton, B. (2013). Perceived barriers to physical activity among pregnant women living in a rural community. Public Health Nursing, 30(4), 361-369.

Mendes, K. D. S., Silveira, R. C. C. P., \& Galvão, C. M. (2008). Revisão integrativa: método de pesquisa para a incorporação de evidências na saúde e na enfermagem. Texto Contexto Enfermagem, 17(3), 758-64.

Mudd, L. M., Nechuta, S., Pivarnik, J. M., \& Paneth, N. (2009). Factors associated with women's perceptions of physical activity safety during pregnancy. Preventive Medicine, 49(2), 194-199. 
Mullan, B., Henderson, J., Kothe, E., Allom, V., Orbell, S., \& Hamilton, K. (2016). The role of habit and perceived control on health behavior among pregnant women. American Journal of Health Behavior, 40(3), 291-301.

Muzigaba, M., Kolbe-Alexander, T. L., \& Wong, F. (2014). The perceived role and influencers of physical activity among pregnant women from low socioeconomic status communities in South Africa. Journal of Physical Activity and Health, 11(7), 1276-1283.

Nascimento, S. L., Godoy, A. C., Surita, F. G., \& Silva, J. L. P. (2014). Recomendações para a prática de exercícios físicos na gravidez: uma revisão crítica da literatura. Revista Brasileira de Ginecologia e Obstetrícia, 36(9), 423-431.

Newham, J.J, Allan, C., Leahy-Warren, P., Carrick-Sen, D., \& Alderdice, F. (2016). Intentions toward physical activity and resting behavior in pregnant women: using the theory of planned behavior framework in a cross-sectional study. Birth, 43(1), 49-57.

Oostdam, N., Van Poppel, M. N. M., Wouters, M. G. A.J., Eekhoff, E. M. W., Bekedam, D. J., Kuchenbecker, W. K. H., ...Van Mechelen, W. (2012). No effect of the FitFor2 exercise program on blood glucose, insulin sensitivity, and birth weight in pregnant women who were overweight and at risk for gestational diabetes: results of a randomized controlled trial. BJOG, 119(9), 1098-1107.

Osuji, T., Lovegreen, S. L., Elliot, M., \& Brownson, R. C. (2006). Barriers to physical activity among women in the rural midwest. Women Health, 44(1), 41-55.

Paul, K. H., \& Olson, C. M. (2013). Moving beyond quantity of participation in process evaluation of an intervention to prevent excessive pregnancy weight gain. Int J Behav Nutr Phys Act, 10(23), 1-4.

Pelaez, M., Gonzalez-Cerron, S., Montejo, R. \& Barakat, R. (2014). Pelvic floor muscle training included in a pregnancy exercise program is effective in primary prevention of urinary incontinence: a randomized controlled trial. Neurourol Urodyn, 33(1), 67-71.

Petherick, E. S. L., Fairley, L., Parslow, R. C., McEachan, R., Tuffnell, D., Pickett, K. E., ... Wright, J. (2016). Ethnic differences in the clustering and outcomes of health behaviors during pregnancy: results from the born in Bradford cohort.J Public Health, 39(3), 514-522.

Poudevigne, M. S., \& O'Connor, P. J. (2006). A review of physical activity patterns in pregnant women and their relationship to psychological health. Sports Med, 36(1), 19-38.

Poudevigne, M. S., \& O'Connor, P. J. (2005). Physical activity and mood during pregnancy. Med Sci Sports Exerc, 37(8), 1374-1380.

Poston, L., Bell, R., Croker, H., Flynn, A. C., Godfrey, K. M., Goff, L., ...Whitworth, M.K. (2015). Effect of a behavioral intervention in obese pregnant women (the UPBEAT study): a multicenter, randomized controlled trial. The Lancet Diabetes \& Endocrinology, 3(10), 767-777.

Robledo-Colonia, A. F., Sandoval-Restrepo, N., Mosquera-Valderrama, Y. F., Escobar-Hurtado, C., \& Ramírez-Vélez, R. (2012). Aerobic exercise training during pregnancy reduces depressive symptoms in nulliparous women: a randomized trial.J Physiother, 58(1), 9-15.

Sangsawang, B. \& Sangsawang, N. (2016). Is a 6-week supervised pelvic floor muscle exercise program effective in preventing stress urinary incontinence in late pregnancy in primigravid women?: a randomized controlled trial. European Journal of Obstetrics \& Gynecology and Reproductive Biology, 197, 103-110.

Santos, P. C., Abreu, S., Moreira, C., Lopes, D., Santos, R., Alves, O., ... Mota, J. (2014). Impact of compliance with different guidelines on physical activity during pregnancy and perceived barriers to leisure physical activity. $J$ Sports Sci, 32(14), 1398-1408.

Sallis, J. F., Owen, N., \& Fisher, E. B. (2008). Ecological models of health behavior. In: Glanz, K., Rimer, B. K., Viswanath, K., organizadores. Health behavior and health education, theory, research, and practice. San Francisco: Jossey-Bass.

Seneviratne, S. N., Jiang, Y., Derraik, J. G. B., McCowan, L. M. E., Parry, G. K., Biggs, J. B., ... Hofmana, P. L. (2016). Effects of antenatal exercise in overweight and obese pregnant women on maternal and perinatal outcomes: a randomised controlled trial. BJOG, 123(4), 588-597.

Shirazian, T., Monteith, S., Friedman, F., \& Rebarber, A. (2010). Lifestyle modification program decreases pregnancy weight gain in obese women. Am J Perinatol, 27(5), 411-414. 
Silva, F. T., Araújo, J. E., Santana, E. F., Lima, J. W., Cecchino, G. N., \& Silva, C. F. D. (2015). Translation and crosscultural adaptation of the Pregnancy Physical Activity Questionnaire (PPAQ) the Brazilian population. Ceska Gynekol, 80(4), 290-298.

Silva Júnior, J. R., Borges, P. S., Agra, K. F., Pontes, I. A., \& Alves, J. G. B. (2013). Effects of an aquatic physical exercise program on glycemic control and perinatal outcomes of gestational diabetes: study protocol for a randomized controlled trial. Trials, 14(1), 390.

Silveira, L. C., Segre, C. A. M. (2012). Physical exercise during pregnancy and its influence in the type of birth. Einstein, $10(4), 409-414$

Stafne, S. N., Salvesen, K. A, Romundstad, P. R., Eggbø, T. M., Carlsen, S. M, \& Mørkved, S. (2012). Regular exercise during pregnancy to prevent gestational diabetes: a randomized controlled trial. Obstetrics \& Gynecology, 119(1), 29-36.

Tavares, J. S., Melo, A. S. O, Amorim, M. M. R., Barros, V. O., Takito, M. Y., Benício, M. H. D. A., \& Cardoso, M. A. A. (2009). Padrão de atividade física entre gestantes atendidas pela estratégia saúde da família de Campina Grande-PB. Rev Bras Epidemiol, 12(1), 10-19.

Torres, R., Soltero, S., Trak, M. A., Tucker, C. M., Mendez, K., Campos, M., ... Palacios, C. (2016). Lifestyle modification intervention for overweight and obese Hispanic pregnant women: development, implementation, lessons learned and future applications. Contemp Clin Trials Commun, 3, 111-116.

Thornton, P. L., Kieffer, E. C., Salabarría-Peña, Y., Odoms-Young, A., Willis, S. K., Kim, H., \& Salinas, M. A. (2006). Weight, diet, and physical activity-related beliefs and practices among pregnant and postpartum latino women: the role of social support. Matern Child Health J, 10(1), 95-104.

Whitford, H. M., \& Jones, M. (2011). An exploration of the motivation of pregnant women to perform pelvic floor exercises using the revised theory of planned behaviour. Br J Health Psychol, 16(4), 761-778.

Whitford, H. M., Alder, B., \& Jones, M. A. (2007). Cross-sectional study of knowledge and practice of pelvic floor exercises during pregnancy and associated symptoms of stress urinary incontinence in North-East Scotland. Midwifery, 23(2), 204- 217.

Wolfe, L. A., Davies, G. A. L. (2003). Canadian guidelines for exercise in pregnancy. Clin Obstet Gynecol, 46(2), 488-495.

Yeo, S., Steele, N. M., Chang, M. C., Leclaire, S. M., Ronis, D. L., \& Hayashi, R. (2000). Effect of exercise on blood pressure in pregnant women with a high risk of gestational hypertensive disorders.J Reprod Med, 45(4), 293-298.

Yeo, S., Cisewski, J., Lock, E. F., \& Marron, J. S. (2010). Exploratory analysis of exercise adherence patterns with sedentary pregnant women. Nurs Res, 59(4), 280.

Yeo, S. (2009). Adherence to walking or stretching, and risk of preeclampsia in sedentary pregnant women. Res Nurs Health, 32(4), 379-390.

Yeo, S., \& Logan, J. G. (2014). Preventing obesity: exercise and daily activities of low-income pregnant women. $J$ Perinatal Neonatal Nurs, 28(1), 17-25.

Weir, Z., Bush, J., Robson, S. C., McParlin, C., Rankin, J., \& Bell, R. (2010). Physical activity in pregnancy: a qualitative study of the beliefs of overweight and obese pregnant women. BMC Pregnancy Childbirth, 10(1), 18.

Whitaker, K. M., Whitaker, K. M., Wilcox, S., Liu, J., Blair, S. N., \& Pate, R. R. (2016). Pregnant women’s perceptions of weight gain, physical activity, and nutrition using Theory of Planned Behavior constructs.J Behav Med, 39(1), 41-54.

Ussher, M., Aveyard, P., Coleman, T., Straus, L., West, R., Marcus, B., Lewis, B., \& Manyonda, I. (2008). Physical activity as an aid to smoking cessation during pregnancy: two feasibility studies. BMC Public Health, 8(1), 328.

Zarvosky, G. S., \& Longo, L. D. (2011). Exercise guidelines in pregnancy: new perspectives. Sports Med, 41(5), 345-360. 